\title{
Use of information technology in emergency and disaster management
}

\author{
Biswajit Mukhopadhyay, Buddhadev Bhattacherjee \\ Faculty: Civil Engineering; Institute: Kolkata Metropolitan Water \& Sanitation Authority, City: Kolkata, India
}

Email address:

biswajitmukherjee23@rediffmail.com (B. Mukhopadhyay), bbudda1234@gmail.com (B. Bhattacherjee)

To cite this article:

Biswajit Mukhopadhyay, Buddhadev Bhattacherjee. Use of Information Technology in Emergency and Disaster Management. American Journal of Environmental Protection. Vol. 4, No. 2, 2015, pp. 101-104. doi: 10.11648/j.ajep.20150402.15

\begin{abstract}
Disaster management being a key part of emergency management encompasses wide range activities. Emergency management programme are developed and implemented through the analysis and information. The majority of information is spatial and can be mapped. GIS allows emergency management to identify onset of any disaster. For this data has to be gathered organized and displayed logically to determine the size and scope of emergency management so that appropriate steps can be taken in case any disaster.
\end{abstract}

Keywords: Manmade, GIS, Preparedness, Mitigation

\section{Introduction}

Disaster management activities in case of a disaster are focused on three primary objects like protecting life, property and environment. Disaster is of two types -

1) Natural and 2) Man- made.

Earthquakes, flood, storm, Hurricane, Tornado, Cyclone, Forest fires etc. are the natural disaster where collapses of high rise buildings, plane crush, fire in building or mal functioning of nuclear facility are the man- made disaster which throw a challenge to the public emergency services for mitigating such disaster efficiently and co-ordinate manner. The optimal provision of information concerning the situation is an essential pre-requisite. Since co-ordination requires current information both from the occurring place and the management groups within a reasonable time which require an integrated communication and information system for disaster management that provides efficient, reliable, secure exchange and processing relevant information.

Disaster management is standing on three major phases like preparedness, prevention and mitigation. Disaster preparedness highlights on warnings and forecasts of impending disasters and often entails processes which are quite dynamic and results in a "rapid onset" disaster. Disaster prevention is a long term activity where satellite monitoring of relevant factors such as changing of land use, population growth is the main criteria. Communication of information about a disaster to a population at risk and appropriate actions to mitigate that hazard is an important part of information technology. The hazard or disaster sometimes cannot be avoided but the negative impacts on the people's property can be mitigated through public warning which is primary goal of the information technology and to ensure to the greatest extend so that hazard may not be a disaster. The success of information technology in case of disaster management is hidden in the seed of communication technology such as evacuation of the people from the hazardous site, to reduce damage to the property and thus minimizing human suffering. It is very important to note that in case of disaster, information technology is not a singular technology to act on but a system constituting the identification, detection, risk assessment of the hazard and accurate identification of the vulnerability of the population at risk and finally communication of information to the vulnerable population about the wide spread activity of the hazards and its rescue measures a sufficient time before so that people can take actions to abort negative consequences to a great extent

\section{Key Players in Disaster Warning}

The following components are considered as the key parties that play major roles in disaster management process-

1. Communities- The communities residing at the bank of the sea or in the hilly area or in the earthquake zone or other disaster prone areas are to be alerted through radio or television messages of on setting any hazard or 
disaster. Community can also be made aware of potential negative impacts to which they are exposed and specific actions to be taken to minimize the threat of loss or damage.

2. Local Government- Local Government should have considerable knowledge of the hazards to which their community is exposed. They must be involved in design and maintenance of early warning systems and able to advice or instruct local population for their safety and reduces the potential loss of resources.

3. National Governments- National Governments are responsible for policies and framework to facilitate the early warnings in addition to the technical system for the preparation and issuance of timely and effective warning to the respective areas of the country. They should ensure the warnings and related responses are directed towards to the most vulnerable population through the design of holistic disaster response and early warnings framework that helps specific needs of related micro and macro level population. Moreover NGO (Non Governmental Organization), private sectors, media and scientific community have a critical and efficient role for mitigation of any natural hazards by supporting scientific and systematic monitoring and warning services to the people's at risk.

\section{Components of Information Technology Used in Disaster Management}

1. Awareness program: To combat disaster, awareness of the risk population about the possible disaster and its combat-strategies is utmost important. It can be done in various ways. If target population are mostly common illiterate people, audio visual aid like Video conferencing, tale- communication even with the experts who are not available at field level can play a great role in educating people to mitigate disaster like situation.

2. Radio \& television - The most traditional electronic media used for disaster warning and a widespread effect to the people. The effectiveness of this media in the developing countries and rural environments where the teledensity is relatively low and there it can be used to spread the warning quickly to a broad population. A study on this media revealed that the effectiveness of radio and television with a easy understandable language of warning can reduce the potential death toll of catastrophic, cyclonic and tidal bore. But only drawback of this system is that at night this media are generally switched off.

3. Telephone/Mobile- The landlines and Mobile phones has an important role in warning the communities at stake for an incoming danger of a disaster. A phone call with warning saved many lives in South Asian Countries in 2004 Tsunami mainly at coastal region.
However there are two drawbacks to warn the people through telephone are -

i. Installation of telephone in the rural areas in all India still is not satisfactory even if there is an exponential use of mobile phone in recent past has mainly bounded in urban areas - particularly in rural and coastal areas the communities are mainly fisherman communities still think that mobile phone is a luxury.

ii. The other drawback is congestion of phone lines before and during the disaster resulting incomplete phone calls at vital period.

4. Short Message Service.(SMS) - the SMS is allowable in most of the mobile phone and permits sending of short messages amount the mobile phone an even land line. In case of failure of network the SMS can work on a different band and can be sent or received even when phone lines are congested. During 2005 Hurricane Katrina disaster in USA, many residence of affected coastal areas were unable to contact relatives and friends through telephone but they could be able to communicate to each other through SMS.

Other communication technologies are used like Cell broadcasting, satellite radio, internet or e-mail, amateur and community radio, etc. to warn the people at stake in case of a impending disaster.

5. Auto- ALERT through Siren /Emergency bell: People in villages who ate not exposed to mobile phones etc can be brought under the auto alert systems of sirens or emergency bells which starts on its own whenever any alert message is detected. They can be installed at offices of local administrations or police stations in the locality.

\section{GIS and Remote Sensing in Disaster Management}

GIS (Geographical Information System) can be defined as a system of hardware and software used for storage, retrieval, mapping and analysis of geographic data. GIS can be used for scientific investigation, resource management and development planning.

Remote Sensing is a measurement of acquisition of information about a phenomenon by a recording device and gathered information about the environment without physical or intimate contact with the object or phenomena.

As disaster management work usually involves different agencies working in different areas. The need for detailed geographical information in order to make critical decision is hike. By utilizing GIS agencies involved in response can share information through data bases on computer generation maps in one location. For disaster management, workers have to access a number of department managers with their unique data and unique maps which in case of a disasters do not allow time to gather these resources. GIS provides the mechanism to centralize and visual display critical information during an emergency. GIS based spaced 
technology solutions have become an integral part of disaster management in developed as well as developing countries.

However incase of failure of GIS system in affected area by thunderstorm or flood like natural disaster, Disaster Management group has to take on physically though data of GIS in pre-failure state can be used to a great extent by the group depending on their expertise and post-failure scenario of GIS. The use of GIS in different phases can be illustrated as follows-

1. Planning

2. Mitigation

3. Preparedness

4. Response

1. Planning- Emergency management in case of a disaster begins with locating and identifying potential emergency problems. The use of GIS can become the backbone of emergency management as it provides a mechanism to centralize and visually display critical information during an emergency. Using a GIS official's can be triggered the bull's eye of the hazards and evaluation of the consequences of potential emergencies or disasters can easily be made. When a disaster like earthquake faults, fire hazards, flood zones, etc are viewed with other map data such as buildings, residential areas, rivers and waterways, streets, pipelines, power lines, etc an official can formulate mitigation, preparedness and possible recovery needs with the help of the information derived from remote sensing and satellite imagery play an importance in disaster management and crisis period. Before an effective emergency management program implementation thorough analysis and planning must be done. GIS facilitates the process by allowing planners to view appropriate combinations of different data to computer generated maps.

2. Mitigation- Mitigation is the activity that actually eliminates or reduces the probability of a disaster by taking preventive measures (e.g. legislation that require stringent building code in earthquake prone area or in case of a flood zones). Mitigation may include the implementation of strict legislation considering the magnitude of an earthquake, characteristics of soil and other geological data, landslide data, vegetation, topography, weather, etc in different areas and take proper action to avoid or to reduce the effects of unavoidable disaster. A GIS can identify certain soil types in an adjacent to an earthquake impact zone whereas bridges and overpass are at risk and GIS can identify the path of a flood based on topographic features or spread of a coastal oil spill based on current and winds. The habited property and human life at risk can easily be identified and targeted for protective actions. Thus based on GIS data safe Zones of habitation can be mapped and accordingly people can be guided properly to choose the safe places.

3. Preparedness- In the preparedness face the Governments developed plans to save lives and minimize the disaster damage. GIS can accurately support better response planning in areas such as determining evacuation routes or locating vulnerable infrastructures and vital lifelines, etc. it also supports logistical planning to be able to provide relief supplies by displaying previously available information on roads, bridges, airports, railways, etc. apart from these activities such as evacuee camp planning can also be done by GIS. GIS can display real time monitoring for emergency early warning. Remote weather stations can provide current weather indexes based on locations and surrounding areas, wind direction, temperature and relative humidity, etc. Wind information is vital in predicting an early report the movement of chemical cloud release or anticipating the direction of wild fire spread upon.

4. Response - Response are the activities followed by a disaster. These activities are designed to provide emergency assistance for victims (e.g. search and rescue emergency shelter, medical care, mass feeding, etc). GIS can provide one of the primary components for Computer aided dispatch (CAD) system. Emergency response units based at fixed locations can be selected and routed for emergency response. The quickest response units can be selected, routed and dispatched to the emergency zone. Depending on the emergency a GIS can provide a detailed information before the first unit arrived. For example during a commercial; building fire the location of the closest hydrants, electrical panels, floor planning of the building can be identified in case of emergency through GIS.

\section{Purification and Treatment of Water During Disaster}

Water is essential requirement for the victims of disasters before during and after their disastrous condition. Storage of water in an hygienic condition is very important. Water management is considered as prerequisite at all stages of disaster management: Preparedness, reduction and mitigation. Several conventional water storage techniques and knowhow have been successfully implemented for combat of the disaster situation for the benefit of people. The disaster management groups use the fresh water to the affected community in both pre and post disaster situation that include (1) storage of water, (2) purification and treatment of water.

(1) Storage of water: In the outbreak of any disaster like Cyclone, Earthquake or any other disaster, the affected community might not have the access to water for days or even for weeks also. Information Technology can play an important role to educate the people of disaster prone area by letting them know technical know-how through public address system like radio, Television, mobile phones to keep the safe storage water in purified condition for a week so that requirement of water for their different house hold purposes during the time of disaster may be adopted.

Containers for water storage: of the many type of containers available for water storage, the glass/ fiber glass, plastic or metal containers are used widely.

Disinfection of water containers: water containers should 
be disinfected before filling them with purified water. In case of disaster the purified and disinfected water are filled up mainly in plastic packets and are distributed to the affected population. In case of house hold disinfection the water can be boiled for 10 minutes for disinfection of water.

Chemical disinfection like sodium hypo-chloride or bleaching powder may be used for disinfection of water in case of outbreak of any disaster.

(2) Purification and treatment of water: In the disaster situation mainly at the time of cyclone, floods or earthquake the water lines and sewerage lines are damaged and they mixed with each other. Then outbreak of many water borne diseases like Cholera, Hepatitis 'A', Dysentery, Typhoid etc can occur due to contamination of water may be treated in various methods like heating, chemical disinfection, filtration or an appropriate combination of these methods. The one method is not sufficient for purification but a series of combination of methods may give full proof disinfected water. Heating or boiling or chlorination are the process which can kill the microbes but other contaminants like dissolve solids, heavy metals. So, for purification purpose, starting from sedimentation for removing suspended particles to filtration through Rapid gravity or slow sand filtration is done for removing dissolved solids. Many advanced method of water purification have been developed and marketed recently which could have potential application in case of disaster situation. In rural area when any outbreak of disaster occur people used two or three earthen jar placed one on top of the other having different diameters of holes starting from $0.5 \mathrm{~mm}$ to $2.0 \mathrm{~mm}$ through which water is passing away and from the bottommost jar the water is taken out and one drop of hypo-chloride solution in 1 liter of water is used for disinfection. Information technology can play a role in mapping of disaster affected areas and help to divide it in several zones based on the information received regarding intensity of disaster in each affected zone and class of people live in that Zone. Accordingly Sectorial action plan is takenwhich suggests what kind of water treatment processes to be adopted for each of the affected Zones. Information technology can further be used in quality control of treatment process by two way communications with the people at field to the experts who are physically miles apart but virtually present.

\section{Conclusion}

In the time of disaster the people get puzzle and during outbreak of any disaster like cyclone, flood or earthquake or any other natural calamities the preparedness and response are very important part of mitigation management. Radio, television, mobile phone are the communication media help us in preparedness and arrangements for mitigation of the disaster situation by storing sufficient food stuff, purified water bottle or plastic pouch are very much essential. Thus the use of Information Technology plays a very important role in case of disaster or emergency management.

\section{References}

[1] Barrett, E.C., Beaumont, M.J. and Herschy, R.W. (1990). "Satellite Remote Sensing for Operational Hydrology: Present Needs and Future Opportunities." Remote Sensing Review, $4(2), 351-466$.

[2] Borneman, R. (1988). "Satellite Rainfall Estimating Program of NOAA/NESDIS Satellite Analysis branch."National Weather Digest, 13(2), 7-15.

[3] Chavez, E., Ide, R., Kirste, T. (1999). Interactive applications of personal situation aware assistants. Computers \& Graphics, 23(6):903-915, 1999.

[4] Guttman, E. (2001). Auto configuration for IP Networking: Enabling Local Communication, IEEE Internet Computing, May/June 2001.

[5] Mobile Broadband for Emergency and Safety Applications: MESA Project, http://www.projectmesa.org/

[6] Barber, D.G., Hochheim, K.P., Dixon, R., Mossocrop, D.R. and McMullan, M.J. (1996). "The role of Earth Observation Technologies in Flood mapping: A Manitoba Case Study". Canadian Journal of Remote Sensing, 22(1), 137-143.

[7] Bruynzeel, L.A. (1990). Hydrology of moist tropical forests and effects of conservation: a state of knowledge review. UNESCO, 224.

[8] Farley, J.A., Hecht, L.G. (1999). Open computing technologies as infrastructures for disaster management. In GDIN conference, Mexico city, May 1999. 